\title{
Diversity of earthworms (Clitellata: Lumbricidae) from Sredna Gora Mountain (Bulgaria)
}

\author{
H. VALCHOVSKI ${ }^{1 *}$ and E. VELIZAROVA ${ }^{2}$ \\ ${ }^{1}$ Hristo Valchovski, Department of Soil Microbiology, Institute of Soil Science, Agrotechnologies and \\ Plant Protection "N. Poushkarov", Agricultural Academy, 7 Shosse Bankya Str., 1080 Sofia, Bulgaria \\ *Corresponding author: e-mail: h_valchovski@abv.bg \\ ${ }^{2}$ Emiliya Velizarova, Department of Forest Ecology, Forest Research Institute, Bulgarian Academy of \\ Sciences, 132, Kl. Ohridski Bld., 1756 Sofia, Bulgaria
}

\begin{abstract}
In the current study the diversity, zoogeographical position and distribution of earthworms from Sredna Gora Mountain (Bulgaria) is presented. During the present investigation, altogether ten earthworm species belonging to seven genera were collected. Among them, seven taxa are reported for the first time from the Sredna Gora Mt.: Cernosvitovia rebeli, Dendrobaena alpina, Allolobophoridella eiseni, Dendrodrilus rubidus rubidus, Aporrectodea caliginosa, Aporrectodea rosea and Lumbricus terrestris. On the basis of the new and literature data here we provide the first list of lumbricid earthworms from Sredna Gora Mountain.
\end{abstract}

Keywords. Soil fauna, Clitellata, earthworms, zoogeography, distribution

\section{INTRODUCTION}

$\mathrm{T}$ he Sredna Gora Mountain is situated in the central part of Bulgaria, bounded on the north by the Sub-Balkan plains and on the south the Thracian valley. The mountain is bordered by the Iskar River to the west and Tundzha River to the east. The Sredna Gora Mountain is some $285 \mathrm{~km}$ long and about $50 \mathrm{~km}$ wide with highest peak Goliam Bogdan $1603 \mathrm{~m}$ a.s.l. The mountain's land area covers $5950 \mathrm{~km}^{2}$ and the average height is $608 \mathrm{~m}$ a.s.l. The Mountain is divided to three parts: Ihtimanska Sredna Gora (western part), Sashtinska Sredna Gora (central part) and Surnena Sredna Gora (eastern part) (Michev et al. 1980).

Earthworm biodiversity in Sredna Gora Mountain is almost unexplored. Its lumbricid fauna hasn't been studied so far; there are only sporadic data from this area. Previous data from Sredna Gora Mountain of Mihailova (1966), Šapkarev (1986) and Valchovski \& Szederjesi (2016) altogether recorded 8 earthworm species and subspecies: Aporrectodea longa (Ude, 1885), Aporrectodea jassyensis (Michaelsen, 1891), Dendro- baena hortensis (Michaelsen, 1890), Dendrodrilus rubidus subrubicundus (Eisen, 1874), Eisenia fetida (Savigny, 1826), Eiseniella tetraedra (Savigny, 1826), Lumbricus rubellus Hoffmeister, 1843 and Octolasion lacteum (Örley, 1881).

The aim of this paper is to summarize the new data as well as the literature records on the earthworm fauna to present the lumbricid diversity of the Sredna Gora Mountain.

\section{MATERIALS AND METHODS}

The field investigations were carried out during the spring of 2016. Earthworms were collected by the diluted formaldehyde method (Raw 1959) complemented with digging and hand-sorting. The combination of both methods provides a more complete sampling of species, because the formalin method alone is not efficient in collecting species living in horizontal burrows. All the specimens were killed in $96 \%$ ethanol, fixed in $4 \%$ formalin solution and in $96 \%$ ethanol, and then transferred into $75 \%$ ethanol. The material is deposited in the Institute of Soil 
Science, Agrotechnologies and Plant Protection "N. Poushkarov", Sofia, Bulgaria in private earthworm collection of Hristo Valchovski (PC $\mathrm{HV})$. Identification of species was done in accordance to Mršić (1991).

The collecting sites are as follows:

1. Bunovo - Ihtimanska Sredna Gora Mt., Mirkovo County, mixed forest roadside east of the village, $907 \mathrm{~m}$.

2. Dolno Kamartsi - Ihtimanska Sredna Gora Mt., Gorna Malina County, mixed forest south of the village, $839 \mathrm{~m}$.

3. Koprivshtitsa - Sashtinska Sredna Gora Mt., coniferous forest north of the village, $980 \mathrm{~m}$.

4. Miromir - Sashtinska Sredna Gora Mt., Hisarya County, pine forest, $365 \mathrm{~m}$.

5. Miromir - Sashtinska Sredna Gora Mt., Hisarya County, oak forest, $345 \mathrm{~m}$.

6. Dolna Banya - Ihtimanska Sredna Gora Mt., north of the village, brook near Maritsa River, $613 \mathrm{~m}$.

7. Dolna Banya - Ihtimanska Sredna Gora Mt., north of the village, oak forest, $677 \mathrm{~m}$.

8. Dolna Banya - Ihtimanska Sredna Gora Mt., north of the village in burned coniferous forest, $679 \mathrm{~m}$.

\section{RESULTS}

\section{Family Lumbricidae Rafinesque-Schmaltz, 1815}

\section{Genus Allolobophoridella Mršić, 1990}

\section{Allolobophoridella eiseni (Levinsen, 1884)}

Lumbricus eiseni Levinsen, 1884: 241.

Eisenia eiseni: Zicsi \& Csuzdi 1986: 114.

Allolobophoridella eiseni: Mršić 1991: 255.

Material examined. PCHV/64 one ex., Mirkovo County, Bunovo, under bark of fallen birch, mixed forest roadside east of the village, $907 \mathrm{~m}, \mathrm{~N}$ $42^{\circ} 42^{\prime} 17$ ', E $23^{\circ} 54^{\prime} 33^{\prime}$, leg. H. Valchovski, 03.04.2016.

\section{Genus Aporrectodea Örley, 1885}

\section{Aporrectodea caliginosa caliginosa (Savigny, 1826)}

Enterion caliginosum Savigny, 1826: 180.
Allolobophora nocturna Evans, 1946: Mihailova 1966: 189 (misidentification).

Allolobophora caliginosa f. typica: Mihailova 1966: 187. Allolobophora caliginosa: Plisko 1963: 429.

Allolobophora caliginosa caliginosa: Šapkarev 1986: 82. Aporrectodea caliginosa caliginosa: Stojanović et al. 2012: 11; Valchovski 2012: 88

Aporrectodea caliginosa: Stojanović et al. 2013: 637.

Material examined. PCHV/69 5 ex., Dolna Banya, brook near Maritsa River, 613m, N $42^{\circ}$ 19' 13”, E $23^{\circ} 46^{\prime}$ 28”, leg. H. Valchovski and E. Velizarova, 13.04.2016.

\section{Aporrectodea jassyensis jassyensis (Michaelsen, 1891)}

Allolobophora jassyensis Michaelsen, 1891: 15; Mihailova 1966: 188; Šapkarev 1986: 83.

Aporrectodea jassyensis jassyensis: Valchovski 2014: 3. Aporrectodea jassyensis: Szederjesi 2013: 77.

Material examined. PHCV/66 2 ex., Hisarya Miromir district, pine forest, $365 \mathrm{~m}, \mathrm{~N} 42^{\circ} 28^{\prime} 47^{\prime \prime}$, E 24 43' 46”, leg. H. Valchovski, 09.04.2016.

\section{Aporrectodea rosea rosea (Savigny, 1826)}

Enterion roseum Savigny, 1826: 182.

Allolobophora prashadi (Stephenson, 1922): Mihailova $1964: 167$.

Eisenia rosea var. typica: Mihailova 1966: 185.

Eisenia rosea var. macedonica (Rosa, 1893): Mihailova 1966: 186.

Eisenia rosea var. bimastoides (Cognetti, 1901): Mihailova 1966: 186.

Allolobophora rosea: Plisko 1963: 428.

Allolobophora rosea rosea: Šapkarev 1986: 81.

Aporrectodea rosea rosea: Valchovski 2014: 3

Aporrectodea rosea: Stojanović et al. 2012: 9.

Material examined. PHCV/66 eight ex., Hisarya Miromir district, pine forest, $365 \mathrm{~m}, \mathrm{~N} 42^{\circ} 28^{\prime}$ 47", E $24^{\circ} 43^{\prime} 46^{\prime}$, leg. H. Valchovski, 09.04. 2016; PCHV/67 three ex., Dolna Banya, north of the village in burned coniferous forest, $679 \mathrm{~m}, \mathrm{~N}$ $42^{\circ}$ 19' 30', E 23 46' 41', leg. H. Valchovski \& E. Velizarova, 13.04.2016; PCHV/68 one ex., Dolna Banya, oak forest, $677 \mathrm{~m}, \mathrm{~N} 42^{\circ} 19^{\prime} 13^{\prime \prime}, \mathrm{E}$ $53^{\circ} 46^{\prime}$ 43", leg. H. Valchovski \& E. Velizarova, 13.04.2016. 


\section{Genus Cernosvitovia Omodeo 1956}

\section{Cernosvitovia rebeli (Rosa, 1897)}

(Figure 1)

Allolobophora rebeli Rosa, 1897: 2.

Octolasium rebeli: Černosvitov 1934: 77; 1937: 89; Mihailova 1966: 194.

Cernosvitovia rebeli: Valchovski 2012: 91; Szederjesi 2013: 78.

Material examined. PCHV/64 one ex., Mirkovo County, Bunovo, mixed forest roadside east of the village, $907 \mathrm{~m}, \mathrm{~N} 42^{\circ} 42^{\prime} 17^{\prime}$, E $23^{\circ} 54^{\prime}$ 33”, leg. H. Valchovski, 03.04.2016; PHCV/66 two ex., Hisarya Miromir district, oak forest, $345 \mathrm{~m}, \mathrm{~N} 42^{\circ} 28^{\prime}$ 55”, E $24^{\circ} 43^{\prime}$ '43”, leg. H. Valchovski, 09.04.2016.

\section{Genus Dendrobaena Eisen, 1873}

\section{Dendrobaena alpina (Rosa, 1884)}

Allolobophora alpina Rosa, 1884: 28.

Eisenia alpina f. typica: Černosvitov 1937: 80; Mihailova 1966: 185.

Dendrobaena alpina: Plisko 1963: 437; Zicsi \& Csuzdi 1986: 118; Valchovski 2012: 91.

Material examined. PCHV/64 one ex., Mirkovo County, Bunovo, mixed forest roadside east of the village, $907 \mathrm{~m}, \mathrm{~N} 42^{\circ} 42^{\prime} 17^{\prime}$, E $23^{\circ} 54^{\prime}$ 33”, leg. H. Valchovski, 03.04.2016; PCHV/63 four ex., Gorna Malina county, Dolno Kamartsi, mixed forest south of the village, $839 \mathrm{~m}, \mathrm{~N} 42^{\circ} 42^{\prime}$ 47”, E $23^{\circ} 52^{\prime}$ 55”, leg. H. Valchovski, 03.04. 2016; PCHV/65 , three ex., Koprivshtitsa, coniferous forest north of the village, $980 \mathrm{~m}, \mathrm{~N} 42^{\circ} 40^{\prime}$ 00”, E $24^{\circ} 20^{\prime} 34$ ”, leg. H. Valchovski, 03.04. 2016; PCHV/67 three ex., Dolna Banya, north of the village in burned coniferous forest, $679 \mathrm{~m}, \mathrm{~N}$ $42^{\circ} 19^{\prime} 30^{\prime \prime}$, E $23^{\circ} 46^{\prime}$ 41”, leg. H. Valchovski \& E. Velizarova, 13.04.2016; PCHV/68 one ex., Dolna Banya, oak forest, $677 \mathrm{~m}, \mathrm{~N} 42^{\circ} 19^{\prime} 13^{\prime \prime}$, E $53^{\circ} 46$ ' 43", leg. H. Valchovski \& E. Velizarova, 13.04.2016.

\section{Genus Dendrodrilus Omodeo, 1956}

\section{Dendrodrilus rubidus rubidus (Savigny, 1826)}

Enterion rubidum Savigny, 1826: 182.
Dendrodrilus rubidus: Perel 1979: 200.

Dendrobaena rubida: Plisko 1963: 434.

Bimastus tenuis: Mihailova 1966: 192.

Dendrobaena rubida tenuis: Zicsi \& Csuzdi 1986: 119. Dendrodrilus rubidus rubidus: Šapkarev 1986: 79.

Material examined. PCHV/64 three ex., Mirkovo County, Bunovo, under bark of fallen birch, mixed forest roadside east of the village, $907 \mathrm{~m}, \mathrm{~N}$ $42^{\circ} 42^{\prime}$ '17', E $23^{\circ}$ 54' 33”, leg. H. Valchovski, 03.04. 2016.

\section{Genus Lumbricus Linnaeus, 1758}

\section{Lumbricus rubellus Hoffmeister, 1843}

Lumbricus rubellus Hoffmeister, 1843: 187; Plisko 1963: 438; Mihailova 1966: 194; Zicsi \& Csuzdi 1986: 120; Šapkarev 1986: 85; Stojanović et al. 2012: 9; Valchovski 2014: 5.

Material examined. PHCV/66 one ex., Hisarya Miromir district, pine forest, $365 \mathrm{~m}, \mathrm{~N} 42^{\circ} 28^{\prime} 47^{\prime}$ ' E 24 43' 46", leg. H. Valchovski, 09.04.2016; PCHV/68 four ex., Dolna Banya, oak forest, $677 \mathrm{~m}, \mathrm{~N} 42^{\circ} 19^{\prime} 13^{\prime \prime}$ E $53^{\circ} 46^{\prime} 43^{\prime}$, leg. H. Valchovski \& E. Velizarova, 13.04.2016.

\section{Lumbricus terrestris Linnaeus, 1758}

Lumbricus terrestris Linnaeus, 1758: 647; Černosvitov 1937: 90; Plisko 1963: 438; Šapkarev 1986: 85; Zicsi \& Csuzdi 1986: 120; Stojanović et al. 2012: 9; Szederjesi 2013: 80; Valchovski 2014: 5.

Material examined. PCHV/65 two ex., Koprivshtitsa, coniferous forest north of the village, $980 \mathrm{~m}, \mathrm{~N} 42^{\circ} 40^{\prime} 00^{\prime}$, E $24^{\circ} 20^{\prime}$ 34”, leg. H. Valchovski, 03.04.2016; PCHV/69 seven ex., Dolna Banya, brook near Maritsa River, 613m, N $42^{\circ} 19^{\prime} 13$ ', E $23^{\circ} 46^{\prime} 28^{\prime}$, leg. H. Valchovski \& E. Velizarova, 13.04.2016.

\section{Genus Octolasion Örley, 1885}

\section{Octolasion lacteum (Örley, 1881)}

Lumbricus terrestris var. lacteum Örley, 1881: 584. Octolasium lacteum: Černosvitov 1934: 76., 1937: 89; Plisko 1963:432; Mihailova 1966: 193; Zicsi \& Csuzdi 1986: 120.

Octolasion tyrtaeum: Mršić 1991: 347. 
Octolasion lacteum: Šapkarev 1986: 84; Szederjesi 2013: 81; Stojanović et al. 2012: 9; Valchovski 2014: 6 .

Material examined. PCHV/63 one ex., large form, Gorna Malina county, Dolno Kamartsi, mixed forest south of the village, $839 \mathrm{~m}, \mathrm{~N} 42^{\circ} 42^{\prime}$ $47^{\prime}$, E $23^{\circ} 52^{\prime}$ 55”, leg. H. Valchovski, 03.04. 2016.

\section{DISCUSSION}

The list of earthworm species and subspecies recorded for Sredna Gora Mountain contains 15 taxa (Table 1). Out of the 10 earthworm species found in the present survey 7 proved to be new to the fauna of the explored area; these are Cernosvitovia rebeli, Dendrobaena alpina, Allolobophoridella eiseni, Dendrodrilus rubidus rubidus, Aporrectodea caliginosa, Aporrectodea rosea and Lumbricus terrestris.

The Balkan endemic species Cernosvitovia rebeli has not been registered previously from this region. In Bulgaria rare Cernosvitovia rebeli was recorded only from a few localities so far: Balkan Mts. (Rosa 1897, Černosvitov 1934), Strandja Mt. (Černosvitov 1937, Szederjesi 2013) and Rhodope Mts. (Mihailova 1966, Szederjesi 2013). Sredna Gora Mountain is situated between the Rhodope Mts. and Balkan Mts. representing the southern and northern localities of Cernosvitovia rebeli respectively (Fig. 1).

According to the zoogeographical composition, the earthworm fauna of the Sredna Gora Mountain is highly peregrine. From the 15 lumbricid species recorded $11(73.3 \%)$ belong to this chorological group. From the four autochthonous species $(26.7 \%)$ C. rebeli is a Balkanic endemism, $D$. alpina possesses a Balkanic-Alpine distribution, Ap. jassyensis is Trans-Aegean and Ai. eiseni has a larger Holarctic range.

It is to be expected that in the future further new lumbricid species will be found in the region, because large areas have not been explored yet including the territory of the wildlife sanctuary of the Sredna Gora Mountain.

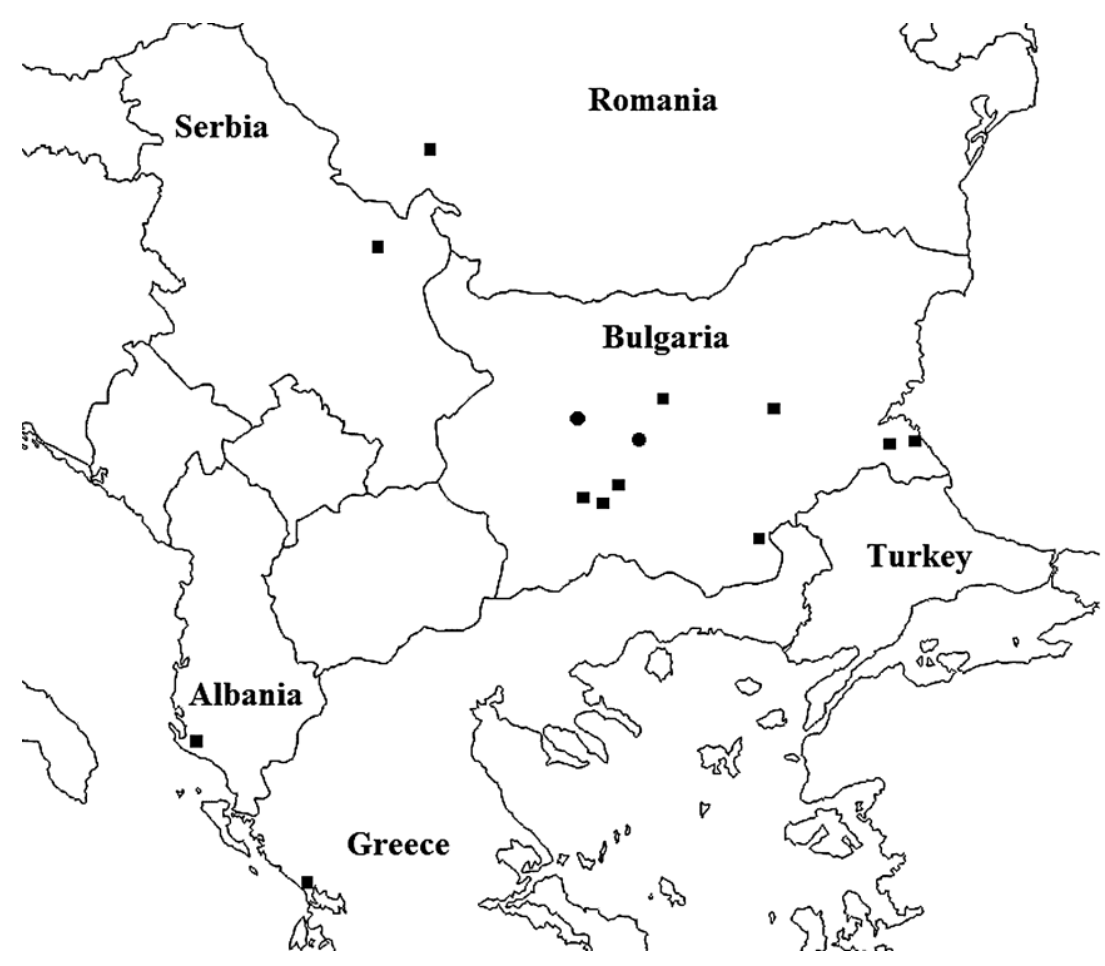

Figure 1. Distribution of Cernosvitovia rebeli (Rosa, 1897) on the Balkan Peninsula. Black dots $=$ new records, black squares $=$ literature data. 
Table 1. List of earthworm species from Sredna Gora Mountain

\begin{tabular}{|l|l|l|}
\hline Earthworm species & Zoogeographical position & Distribution in Sredna Gora Mt. \\
\hline Allolobophoridella eiseni & Holarctic & Ihtimanska Sredna Gora \\
\hline Aporrectodea caliginosa & Peregrine & Ihtimanska Sredna Gora \\
\hline Aporrectodea jassyensis & Trans-Aegean & Sashtinska Sredna Gora \\
\hline Aporrectodea longa & Peregrine & Surnena Sredna Gora \\
\hline Aporrectodea rosea & Peregrine & Sashtinska and Ihtimanska Sredna Gora \\
\hline Cernosvitovia rebeli & Endemic & Sashtinska and Ihtimanska Sredna Gora \\
\hline Dendrobaena alpina & Balkanic-Alpine & Sashtinska and Ihtimanska Sredna Gora \\
\hline Dendrobaena hortensis & Peregrine & Surnena Sredna Gora \\
\hline Dendrodrilus rubidus rubidus & Peregrine & Ihtimanska Sredna Gora \\
\hline Dendrodrilus rubidus subrubicundus & Peregrine & Surnena Sredna Gora \\
\hline Eisenia fetida & Peregrine & Sashtinska Sredna Gora \\
\hline Eiseniella tetraedra & Peregrine & Surnena and Sushtinska Sredna Gora \\
\hline Lumbricus rubellus & Peregrine & Sashtinska and Ihtimanska Sredna Gora \\
\hline Lumbricus terrestris & Peregrine & Sashtinska and Ihtimanska Sredna Gora \\
\hline Octolasion lacteum & Peregrine & Ihtimanska and Surnena Sredna Gora \\
\hline
\end{tabular}

\section{REFERENCES}

Bouche, M.B. (1972): Lombriciens de France, écologie et systématique. I.N.R.A. Publications 72-2. Institut National de la Recherche Agronomique, Paris, $621 \mathrm{pp}$.

ČERnOSVITOV, L. (1934): Die Lumbriciden Bulgariens. Mitteilungen aus den Königlich Naturwissenschaftlichen Instituten in Sofia, 7: 71-78.

ČERnOSVITOV, L. (1937): Die Oligochaetenfauna Bulgariens. Mitteilungen aus den Königlich Naturwissenschaftlichen Instituten in Sofia, 10: 62-92.

EISEN, G. (1873): Om Skandinaviens Lumbricider. Öfversigt af Kongliga Vetenskaps-Akademiens Förhandligar, 30(8): 43-56.

EISEN, G. (1874): New Englands och Canadas lumbricides. Öfversigt af Kongliga Vetenskaps-Akademiens Förhandlingar Stockholm 31 (2): 41-49.

HofFMEISTER, W. (1843): Beitrag zur Kenntniss deutscher Landanneliden. Archiv für Naturgeschichte, 9: 183-198.

LEVINSEN, G.M.R. (1884): Systematisk-geografisk oversigt over de nordiske Annulata, Gephyrea, Chaetognathi og Balanoglossi. Videnskabelige Meddelelser fra den naturhistoriske Forening $i$ Kjöbenhavn, 45: 92-384.

LinNAEUS, C. (1758): Systema Naturae per Regna tria Naturae, secundum Classes, Ordines, Genera, Species, cum Characteribus, Differentiatiis, Syno- nymis, Locis. 10th edition, volume 1. Laurentii Salvii, Holmiae, 824 pp. doi: 10.5962/bhl.title.542

Michaelsen, W. (1890): Die Lumbriciden NordDeutschlands. Jahrbuch der Hamburgischen Wissenschaftlichen Anstalten, 7: 1-19.

MiCHAELSEN, W. (1891): Oligochaeten des Naturhistorischen Museums in Hamburg IV. Jahrbuch der Hamburgischen Wissenschaftlichen Anstalten, 8: $1-42$.

MicheV, N., Mihailov, C., VAPCARov, I. \& KirADZIEV, S. (1980): Geographical dictionary of Bulgaria. Sofia, Bulgaria. Nauka i izkustvo, Sofia, 561 pp.

Minailova, P. (1964): Njakoj vidove ot semejstvo Lumbricidae (Oligohaeta) novi za faunata na Blgarija. Annuaire de Université de Sofia, 57: 163169.

Minailova, P. (1966) Dzdovni cervi Lumbricidae (Oligohaeta) v Trakija. Fauna na Trakja, Bulgarian Academy of Science, Sofia, 3: 181-200.

MRŠIĆ, N. (1990): Description of a new subgenus, three new species and taxonomic problems of genus Allolobophora sensu Mršić, N. \& Šapkarev, J. 1988 (Lumbricidae: Oligochaeta). Biološki Vestnik, 38 (1): 49-68.

MRŠIĆ, N. (1991): Monograph on earthworms (Lumbricidae) of the Balkans I-II. Slovenska Akademija Znanosti in Umetnosti, Zazred za Naravoslovne Vede Opera 31 Ljubljana, 757 pp. 
OMODEO, P. (1956): Contributo alla revisione dei Lumbricidae. Archivio Zoologico Italiano, 41: 129212.

ÖRLEY, L. (1881): A magyarországi Oligochaeták faunája. I. Terricolae. Mathematikai és Természettudományi Közlemények, 16: 562-611.

ÖRLEY, L. (1885:) A palaearktikus övben élö Terrikoláknak revíziója és elterjedése. Értekezések a Természettudományok Köréböl, 15: 1-34.

PEREL, T.S. (1979): Razprostranenie i zakonomernosti raspredelenia dozdevyh cervej fauny SSSR. Nauka, Moskva, 268 pp.

PlisKO, D. (1963): Materialien zur Kenntnis der Regenwürmer (Oligochaeta, Lumbricidae) Bulgariens. Fragmenta Faunisica, Warsawa, 10: 425-440.

RAFINESQUE-SchMALTZ, C. (1815): Analyse de la Nature ou tableau de l'univers et des corps organisés. Palermo, 223 pp.

RAw, F. (1959): Estimating earthworm population by using formalin. Nature, 184: 1661-1662. doi: $10.1038 / 1841661 \mathrm{a} 0$

RosA, D. (1884): Lumbricidi del Pieomte. Unione Tipografico-Editrice, Torino, $54 \mathrm{pp}$.

RosA, D. (1897): Nuovi lombrichi dell'Europa orienttale. (Seconda serie.) Bollettino dei Musei di Zoologia ed Anatomia comparata della R. Università di Torino, 12(269): 1-5.

ŠAPKAREV, J. (1986): Earthworm fauna of Bulgaria (Oligochaeta: Lumbricidae). Fragmenta Balcanica, 13: 77-94.

SAVIGNY, J.C. (1826): Analyse des Travaux de l'Academie royale des Sciences, pendant l'année 1821, partie physique. In. CUVIER, G. (Ed.) Mémoires de
l'Académie des Sciences de l'Institut de France Paris, 5: 176-184.

Stojanović, M., Tsekova, R. \& Milutinović, T. (2012): Earthworms (Oligochaeta: Lumbricidae) of Bulgaria: Diversity and Biogeographical Review. Acta Zoologica Bulgarica, Suppl., 4: 7-15.

Stojanović, M., Tsekova, R., Pesić, S., Milanović, J. \& MiLutinOViĆ, T. (2013): Diversity and a biogeographical review of the earthworms (Oligochaeta: Lumbricidae) of the Balkan Mountains (Stara Planina Mountains) in Serbia and Bulgaria. Turkish Journal of Zoology, 37: 635-642. doi: 10.3906/zoo-1301-33

SZEDERJESI, T. (2013): New earthworm records from Bulgaria (Oligochaeta, Lumbricidae). Opuscula Zoologica, Budapest, 44: 77-83.

UDE, H. (1885): Über die Rückenporen der terricolen Oligochaeten, nebst Beiträgen zur Histologie des Leibesschlauches und zur Systematik der Lumbriciden. Zeitschrift für Wissenschaftliche Zoologie, 43: $87-143$

VALCHOVSKI, H. (2012): Checklist of earthworms (Oligochaeta: Lumbricidae) from Bulgaria - a review. Zootaxa, 3458: 86-102.

VALCHOVSKI, H. (2014): Diversity of earthworms (Oligochaeta: Lumbricidae) in Sofia Plain, Bulgaria. Zoonotes, 59: 1-9.

VAlCHOVsKi, H. \& SZEDERJESI, T. (2016): New and additional records of earthworms (Oligochaeta: Lumbricidae) from Bulgaria: first finding place of endemic species Cernosvitovia munteniana on the Balkan Peninsula. North-Western Journal of Zoology (in press).

ZICSI, A. \& CSUZDI, Cs. (1986): Regenwürmer aus Bulgarien (Oligochaeta Lumbricidae). Opuscula Zoologica Budapest, 22: 113-121. 